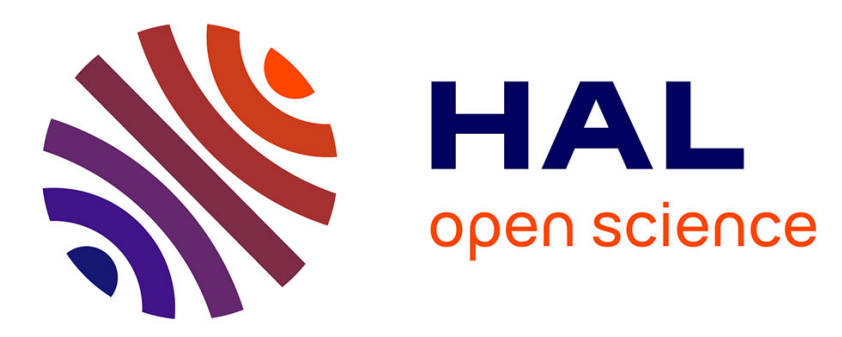

\title{
Effet hall dans les solutions électrolytiques diluées, solvatation et dynamique des ions
}

\author{
M. Meton, P. Gerard, E.J. Picard
}

\section{To cite this version:}

M. Meton, P. Gerard, E.J. Picard. Effet hall dans les solutions électrolytiques diluées, solvatation et dynamique des ions. Journal de Physique Lettres, 1976, 37 (10), pp.247-250. 10.1051/jphyslet:019760037010024700 . jpa-00231285

\section{HAL Id: jpa-00231285 https://hal.science/jpa-00231285}

Submitted on 1 Jan 1976

HAL is a multi-disciplinary open access archive for the deposit and dissemination of scientific research documents, whether they are published or not. The documents may come from teaching and research institutions in France or abroad, or from public or private research centers.
L'archive ouverte pluridisciplinaire HAL, est destinée au dépôt et à la diffusion de documents scientifiques de niveau recherche, publiés ou non, émanant des établissements d'enseignement et de recherche français ou étrangers, des laboratoires publics ou privés. 
Classification

Physics Abstracts

9.220

\title{
EFFET HALL DANS LES SOLUTIONS ÉLECTROLYTIQUES DILUÉES, SOLVATATION ET DYNAMIQUE DES IONS
}

\author{
M. METON, P. GERARD \\ Université Pierre-et-Marie-Curie, U.E.R. de Physique Théorique, \\ Tour 16, $1^{\text {er }}$ étage, 4, place Jussieu, 75320 Paris, France
}

et E. J. PICARD

Laboratoire de Physique des Liquides, Faculté des Sciences, 29283 Brest Cedex, France

(Reçu le 24 mai 1976, accepté le 15 juillet 1976)

\begin{abstract}
Résumé. - Nous présentons dans cette lettre le premier ensemble de mesures cohérentes de la constante de Hall de solutions électrolytiques diluées : 25 acides, bases et sels constitués de 6 anions et 6 cations. Ces résultats sont interprétés en termes de modèles simples de la solvatation ionique.

Abstract. - This letter presents the first collection of consistant measurements of Hall constants for dilute solutions of electrolytes : 25 acids, bases and salts composed of 6 anions and 6 cations. These results are interpreted in terms of simple models for the ionic solvation.
\end{abstract}

1. Introduction. - L'effet Hall est couramment utilisé dans les recherches sur l'état solide, car il permet de déterminer dans certains cas la concentration des porteurs de charge. Son application à l'étude des milieux électrolytiques n'aurait pas le même objectif, puisque la concentration des ions dans la solution est fixée par l'expérimentateur. La connaissance de la constante de Hall fournirait alors des informations sur la mobilité des ions et sur la structure de l'ion solvaté. Dans une expérience de Hall, en effet, le porteur de charge est sollicité dans deux directions perpendiculaires : celle du champ électrique appliqué et celle du champ de Hall qui résulte de la force de Lorentz engendrée par le champ magnétique. On pourrait ainsi mettre en évidence l'existence éventuelle d'une anisotropie de l'ion solvaté.

La mise en œuvre de l'effet Hall dans les milieux liquides s'est heurtée jusqu'ici à des obstacles de nature expérimentale : l'extrême petitesse de l'effet attendu (une théorie élémentaire laisse prévoir des f.e.m. de Hall de l'ordre du $\mu \mathrm{V}$, dans les conditions expérimếntăles-ùsuelles) et le risque d'apparition de mouvements hydrodynamiques en sont des exemples parmi d'autres. L'utilisation d'une cellule de faible épaisseur et de la méthode de la double fréquence [1], un courant alternatif (de $243 \mathrm{~Hz}$ ) et un champ alternatif (de $75 \mathrm{~Hz}$ ) qui engendrent une tension de Hall $(243-75=168 \mathrm{~Hz})$, nous ont permis de surmonter les différents obstacles existants, et nous avons pu mesurer la constante de Hall de 25 électrolytes 1-1 en solution aqueuse, à la température ambiante.
On trouvera dans cette lettre des indications relatives à l'aspect expérimental de notre travail, ainsi que les résultats obtenus. Nous proposerons enfin une interprétation de ces résultats à l'échelle microscopique.

2. Aspect expérimental. - Les valeurs efficaces du courant et du champ magnétique sont respectivement de $500 \mu \mathrm{A}$ et $0,2 \mathrm{~T}$. La cellule utilisée est en silice pure, aux faces polies optiquement, et pourvue d'électrodes de $\mathrm{Pt}$; la lame d'électrolyte a pour dimensions : épaisseur : 0,200 $\mathrm{mm}$; largeur : $8 \mathrm{~mm}$; longueur : $3,5 \mathrm{~cm}$.

Dans le cas le plus favorable, la tension de Hall $V_{H}$ s'élève à environ $250 \mathrm{nV}$. En appliquant sur les électrodes de courant une tension de $250 V_{\text {eff }}$, soit une tension $10^{9}$ fois supérieure à $V_{\mathrm{H}}$, il apparaît une tension de non-alignement $10^{6}$ fois supérieure à $V_{\mathrm{H}}$. La tension induite par le champ magnétique dans les différentes boucles des circuits de mesure et de courant s'élève à quelques centaines de $\mathrm{mV}$ et plus si aucune précaution n'est prise. Ces deux tensions parasites engendrent alors dans les éléments non linéaires des différents circuits des tensions d'intermodulation à la fréquence de Hall, qui faussent radicalement les mesures. Pour obtenir des résultats reproductibles il faut annuler presque entièrement, par un jeu de contre-boucles, la tension induite dans chacun des circuits. La détection du signal de Hall s'effectue en mode différentiel. L'adaptateur d'impédance est un préamplificateur différentiel (PAR 123). 


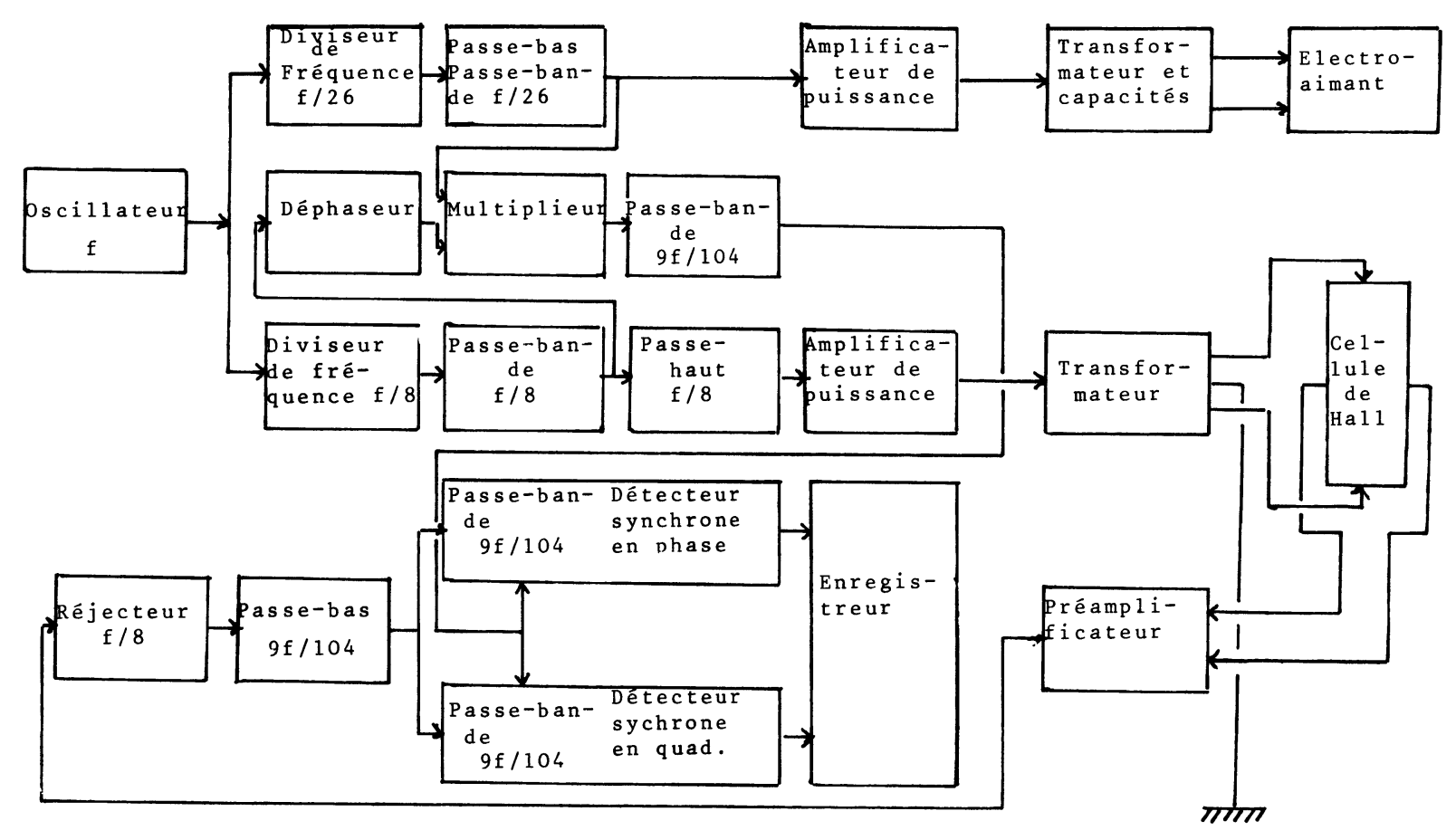

ORGAN IGR AMME

Le signal obtenu est ensuite envoyé dans un filtre rejeteur du $243 \mathrm{~Hz}$, puis dans un amplificateur sélectif passe-bas, avant d'être injecté dans un détecteur synchrone (Lock in amplifier PAR 124) qui donne directement $V_{\text {Heff. }}$ La tension de référence qui pilote le détecteur synchrone est obtenue à partir d'un signal unique envoyé dans des diviseurs de fréquence et un multiplieur. A l'aide du déphaseur la phase de cette référence est réglée de manière à être la même que celle du signal de Hall attendu. Notre dispositif nous permet donc de déterminer le signe de la constante de Hall $R_{\mathrm{H}}$.

3. Résultats. - En première approximation [2-4],

$$
R_{\mathrm{H}}=(1 / n e) \frac{\mu_{+}-\left|\mu_{-}\right|}{\mu_{+}+\left|\mu_{-}\right|}
$$

où $\mu_{+}$et $\mu_{-}$sont les mobilités électriques des porteurs de charge, $n$ leur densité volumique, $e$ la charge élémentaire. En unités S I, $R_{\mathrm{H}}$ s'exprime en $\mathrm{m}^{3} \cdot \mathrm{A}^{-1} \cdot \mathrm{s}^{-1}$. L'approximation consiste à prendre $\mu_{\mathrm{e}}\left(\mathbf{v}_{\mathrm{e}}=\mu_{\mathrm{e}} \mathbf{E}\right)$ égale à la mobilité électromagnétique $\mu_{\mathrm{m}}$ définie par $\mathbf{v}_{\mathbf{H}}=\mu_{\mathrm{m}} \mathbf{E}_{\mathrm{H}}$ (où $\mathbf{E}_{\mathrm{H}}=\mathbf{v}_{\mathrm{e}} \wedge \mathbf{B}$ ), $\mathbf{v}_{\mathrm{e}}$ et $\mathbf{v}_{\mathbf{H}}$ étant les vitesses moyennes des porteurs de charge dans les champs $\mathbf{E}$ et $\mathbf{E}_{\mathbf{H}}$. Appelons nombres ioniques de Hall les rapports $h_{-}=\mu_{\mathrm{m}^{-}} / \mu_{\mathrm{e}^{-}}$et $h_{+}=\mu_{\mathrm{m}^{+}} / \mu_{\mathrm{e}^{+}}$; (1) correspond alors à $h_{+}=h_{-}=1$.

Les résultats obtenus sont donnés dans le tableau I. En pratique, le signal de Hall est enregistré et ses valeurs mesurées toutes les $10 \mathrm{~s}$ pendant un temps qui va de quelques minutes à plus de $30 \mathrm{mn}$, et ces mesures répétées $n$ fois fournissent la valeur moyenne $V_{\mathrm{H}}$ retenue, $\Delta V_{\mathrm{H}} \simeq 20 \mathrm{nV}$.
TABLEAU I

\begin{tabular}{|c|c|c|c|c|c|c|}
\hline & $\mathrm{OH}^{-}$ & $\mathrm{F}^{-}$ & $\mathrm{Cl}^{-}$ & $\mathrm{Br}^{-}$ & $\mathrm{I}^{-}$ & $\mathrm{BPh}^{-}$ \\
\hline $\mathrm{H}^{+}$ & & & $\begin{array}{c}1 \\
+206 \\
+5,83 \\
+0,056 \\
+0,643 \\
+0,058 \\
\end{array}$ & 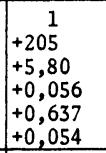 & $\begin{aligned} & 1 \\
&+ 207 \\
&+5,85 \\
&+0,056 \\
&+0,642 \\
&+0,056 \\
&\end{aligned}$ & \\
\hline $\mathrm{Li}^{+}$ & $\begin{array}{l}2,4 \\
-133 \\
-3,76 \\
-0,087 \\
-0,687 \\
-0,092 \\
\end{array}$ & $\begin{aligned} & 3,8 \\
&+ 38 \\
&+1,07 \\
&+0,039 \\
&-0,185 \\
&+0,037 \\
&\end{aligned}$ & $\begin{array}{l} \\
4,19 \\
-89 \\
-2,52 \\
-0,102 \\
-0,339 \\
-0,109 \\
\end{array}$ & $\begin{array}{l}4,05 \\
-140 \\
-3,96 \\
-0,155 \\
-0,348 \\
-0,140 \\
\end{array}$ & $\begin{array}{l} \\
4,24 \\
-115 \\
-3,25 \\
-0,133 \\
-0,342 \\
-0,134 \\
\end{array}$ & \\
\hline $\mathrm{Na}^{+}$ & $\begin{array}{l}1,92 \\
-154 \\
-4,36 \\
-0,081 \\
-0,605 \\
-0,073\end{array}$ & $\begin{array}{l}\quad 3,65 \\
+98 \\
+2,77 \\
+0,096 \\
-0,052 \\
+0,087\end{array}$ & $\begin{array}{l}\quad 4,04 \\
-50 \\
-1,44 \\
-0,055 \\
-0,213 \\
-0,043\end{array}$ & $\begin{array}{l}3,05 \\
-80 \\
-2,26 \\
-0,067 \\
-0,225 \\
-0,077\end{array}$ & $\begin{aligned} & 3,85 \\
&-77 \\
&-2,18 \\
&-0,082 \\
&-0,218 \\
&-0,070\end{aligned}$ & 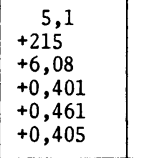 \\
\hline $\mathrm{K}^{+}$ & $\begin{array}{l}1,85 \\
-71 \\
-2,00 \\
-0,037 \\
-0,466 \\
-0,037 \\
\end{array}$ & $\begin{array}{r}3,6 \\
+157 \\
+4,44 \\
+0,154 \\
+0,144 \\
+0,171 \\
\end{array}$ & $\begin{aligned} & 3,40 \\
&+ 67 \\
&+1,90 \\
&+0,055 \\
&-0,020 \\
&+0,053\end{aligned}$ & 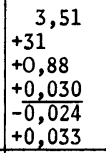 & $\begin{aligned} & 3,12 \\
&+ 56 \\
&+ 1,58 \\
&+ 0,048 \\
&-0,024 \\
&+0,033 \\
&\end{aligned}$ & \\
\hline $\mathrm{Cs}^{+}$ & & & $\begin{array}{l}\quad 3,34 \\
+67 \\
+1,90 \\
+0,060 \\
+0,002 \\
+0,063 \\
\end{array}$ & & $\begin{aligned} & 3,17 \\
&+ 57 \\
&+ 1,61 \\
&+ 0,049 \\
&-0,002 \\
&+0,045 \\
&\end{aligned}$ & \\
\hline $\mathrm{NBu}_{4}{ }^{+}$ & $\begin{array}{l}2,03 \\
-220 \\
-6,22 \\
-0,122 \\
-0,832 \\
-0,132 \\
\end{array}$ & & $\begin{aligned} & 4,68 \\
&-218-6 \\
&-6,17 \\
&-0,279 \\
&-0,620 \\
&-0,285 \\
&\end{aligned}$ & \begin{tabular}{l}
\multicolumn{1}{l}{4,78} \\
-260 \\
$-7,35$ \\
$-0,339$ \\
$-0,627$ \\
$-0,335$ \\
\end{tabular} & 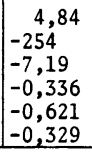 & \\
\hline CLE & 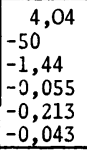 & \multicolumn{5}{|c|}{ 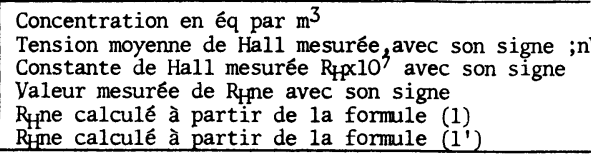 } \\
\hline
\end{tabular}

Les valeurs mesurées $R_{\mathrm{Hm}}$ sont toutes différentes des $R_{\mathrm{Hc}}$ calculées à l'aide de la formule (1), sauf pour $\mathrm{Na}(\mathrm{BPh} 4)$ pour lequel $R_{\mathrm{Hm}} / R_{\mathrm{Hc}}=r \simeq 1$. $\mathrm{Par}$ 
exemple $r \simeq 1 / 12$ pour $\mathrm{HCl}, \mathrm{HBr}$, $\mathrm{HI}$. Pour $\mathrm{KCl}$, la valeur numérique et le signe de $r$ changent : $r \simeq-3$; il en est de même pour $\mathrm{KBr}, \mathrm{KI}$ et $\mathrm{LiF}$ (valeurs soulignées du tableau I).

Pour rendre compte des valeurs expérimentales de $R_{\mathrm{H}}$, nous proposons l'expression :

$$
R_{\mathrm{H}}=(1 / n e) \frac{\mu_{+\mathrm{e}} \mu_{+\mathrm{m}}-\left|\mu_{-\mathrm{m}} \mu_{-\mathrm{e}}\right|}{\left(\mu_{+\mathrm{e}}+\left|\mu_{-\mathrm{e}}\right|\right)^{2}}
$$

que l'on peut réécrire en utilisant les nombres de transport $t$ et les nombres de Hall $h$ :

$$
R_{\mathrm{H}}=(1 / n e)\left(t_{+}{ }^{2} h_{+}-t_{-}{ }^{2} h_{-}\right) .
$$

Si l'on prend $\mu_{\mathrm{m}}=\mu_{\mathrm{e}}$ pour $\mathrm{NBu} 4$ et $\mathrm{BPh} 4$, les nombres de Hall calculés sont donnés dans le tableau II. Les valeurs de $h$ obtenues par un calcul d'optimisation sur ordinateur, qui tient compte des variations de $h$ et de $t$ avec la concentration mais n'impose aucune valeur aux mobilités, sont les mêmes que celles du tableau II aux erreurs d'expérience près.

\begin{tabular}{|c|c|c|c|c|c|c|}
\hline & $\mathrm{H}^{+}$ & $\mathrm{Li}^{+}$ & $\mathrm{Na}^{+}$ & $\mathrm{K}^{+}$ & $\mathrm{Cs}^{+}$ & $\mathrm{NBu}_{4}+$ \\
\hline $\mathrm{h}_{+}$ & 0,1 & 1 & 0,9 & 0,8 & 0,7 & 1 \\
\hline & $\mathrm{OH}^{-}$ & $\mathrm{F}^{-}$ & $\mathrm{C} 1^{-}$ & $\mathrm{Br}^{-}$ & $\mathrm{I}^{-}$ & $\mathrm{BPh}_{4}^{-}$ \\
\hline h. & 0,2 & $\overline{0,4}$ & 0,5 & 0,6 & 0,6 & 1 \\
\hline
\end{tabular}

\section{TABLEAU II}

4. Interprétation. - Nous avons représenté figure 1 les valeurs de $h$ en fonction des rayons ioniques des ions. Les points correspondant aux anions et aux cations des solutions salines sont d'autant plus distants que les rayons cristallins sont plus petits. On ne peut expliquer ce résultat qu'en admettant que l'hydratation des petits anions et cations de même taille est différente. Ce qui peut provenir de ce que l'orientation dans l'espace, le nombre et la durée de vie des liaisons ion-dipôle dépendent du signe de l'ion.

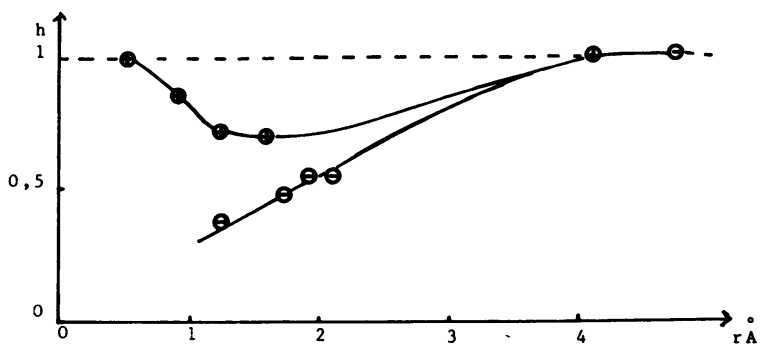

FIG. 1.

Les résultats obtenus, à savoir que les valeurs de $h$ sont inférieures à 1 , peuvent recevoir l'interprétation suivante à l'échelle microscopique.

D'un point de vue phénoménologique, on peut imaginer qu'un ellipsoïde de révolution des mobilités est attaché à chaque porteur, et que l'orientation de chacun de ces ellipsoïdes est la même que celle de la vitesse instantanée de l'ion correspondant. Si, en première approximation, on ne tient pas compte de la déformation de l'ellipsoïde avec l'énergie, on a :

$\mu_{\mathrm{e}}=\left(\mu_{1}+2 \mu_{\mathrm{t}}\right) / 3$ et $\mu_{\mathrm{m}} \mu_{\mathrm{e}}=\left(2 \mu_{1} \mu_{\mathrm{t}}+\mu_{\mathrm{t}}^{2}\right) / 3$

l'ensemble étant en moyenne isotrope, $\mu_{1}$ et $\mu_{\mathrm{t}}$ étant les mobilités longitudinale et transversale du porteur. Si l'on calcule $h$ à partir des expressions (2), on trouve :

$$
h=1-\left\{\left(\mu_{1}-\mu_{\mathrm{t}}\right) / 3 \mu_{\mathrm{e}}\right\}^{2} .
$$

Du point de vue de la mécanique statistique, admettons que le mouvement de l'ion obéit à l'équation de Langevin généralisée :

$$
\dot{p}(t)=-\int_{0}^{t} K(s) p(t-s) \mathrm{d} s+R(t)+X(t),
$$

où $K(t)$ est la fonction de mémoire. Si nous prenons pour $K(t)$ l'expression :

$$
K(t)=\left\{\left(z^{-1}+z\right) / \tau\right\}^{2} \exp ^{-}\left\{\left(z^{-1}+z\right)^{2} t / \tau\right\}
$$

où $z$ est un complexe défini sur le contour ci-contre,

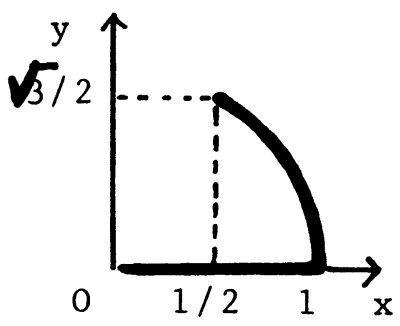

alors la fonction de corrélation normalisée de la vitesse est :

$$
\begin{aligned}
\varphi(t) & =\frac{\langle v(t) \cdot v(0)\rangle_{0}}{\left\langle v(0)^{2}\right\rangle_{0}}=. \\
& =\left(1 / 1-z^{2}\right)\left\{\exp \left[\left(z^{-2}-z^{2}\right) t / 2 \tau\right]\right. \\
- & \left.z^{2} \exp -\left[\left(z^{-2}-z^{2}\right) t / 2 \tau\right]\right\} \exp -\left(z^{-1}+z\right)^{2} t / 4 \tau .
\end{aligned}
$$

Il est alors possible de calculer $\mu_{\mathrm{e}}$ et $h$ comme H. L. Friedman l'a fait [5].

Si l'on utilise les relations :

$$
\mu_{\mathrm{e}}=(e / m) \int_{0}^{\infty} \varphi(t) \mathrm{d} t
$$

et

$$
\mu_{\mathrm{e}} \mu_{\mathrm{m}}=\left(e^{2} / m^{2}\right) \int_{0}^{\infty} t \varphi(t) \mathrm{d} t
$$

le calcul donne :

$$
h=1-1 /\left(z^{-1}+z\right)^{2}
$$


avec

$$
\mu_{\mathrm{e}}=e \tau / m \text {. }
$$

En combinant (4) et (5) avec les relations :

et

$$
\tau=\left(\mu_{1}+2 \mu_{\mathrm{t}}\right) /(3 e / m)
$$

$$
\left(z^{-1}+z\right)^{-1}=\left(\mu_{1}-\mu_{\mathrm{t}}\right) /\left(\mu_{1}+2 \mu_{\mathrm{t}}\right),
$$

on retrouve les équations (2) et (3).

On peut envisager, comme le suggère H. L. Friedman [5] pour $\mathrm{Na}^{+}$et $\mathrm{H}^{+}$, que certains ions existent dans deux états que l'on qualifiera de rapide et de lent, leur durée de vie dans chacun de ces états étant suffisamment longue par rapport au temps moyen entre deux chocs successifs avec les molécules du solvant. La mobilité électrique de tels ions est donnée par $\mu_{\mathrm{e}}=x_{\mathrm{L}} \mu_{\mathrm{eL}}+x_{\mathrm{R}} \mu_{\mathrm{eR}}$ et la mobilité électromagnétique par :

$$
h \mu_{\mathrm{e}}=\frac{x_{\mathrm{L}} h_{\mathrm{L}} \mu_{\mathrm{eL}}^{2}+x_{\mathrm{R}} h_{\mathrm{R}} \mu_{\mathrm{eR}}^{2}}{\mu_{\mathrm{e}}}
$$

$x_{\mathrm{L}}$ et $x_{\mathrm{R}}$ étant les fractions ioniques des particules lentes et rapides $\left(x_{\mathrm{L}}+x_{\mathrm{R}}=1\right)$.

Ainsi, quand :

$h \simeq 1$ : cas des gros ions $\mathrm{NBu}^{+}, \mathrm{BPh}^{-}$ou des petits cations fortements hydratés, $\mathrm{Li}^{+}, \mathrm{Na}_{\mathrm{L}}^{+}$, on a

$$
K(t) \simeq(1 / \tau) \delta(t), \quad \varphi(t) \simeq \mathrm{e}^{-(t / \tau)},
$$

$h \simeq 3 / 4:$ cas de $\mathrm{K}^{+}, \mathrm{Cs}^{+}, \mathrm{Na}_{\mathrm{R}}^{+}, \mathrm{H}_{\mathrm{L}}^{+}$, on $\mathrm{a}:$

$$
\begin{gathered}
K(t) \simeq\left(4 / \tau^{2}\right) \exp -(4 t / \tau), \\
\varphi(t) \simeq(1+2 t / \tau) \exp -(2 t / \tau)
\end{gathered}
$$

$h \simeq 1 / 2$ : c'est approximativement le cas des halogènes et, peut-être, celui de $\mathrm{OH}_{\mathrm{L}}^{-}$, on a :

$$
\begin{gathered}
K(t) \simeq\left(2 / \tau^{2}\right) \exp -(2 t / \tau), \\
\varphi(t) \simeq\{\cos (t / \tau)+\sin (t / \tau)\} \exp -(t / \tau),
\end{gathered}
$$

$h \simeq 0$ : ce serait le cas de $\mathrm{H}_{\mathrm{R}}^{+}$et $\mathrm{OH}_{\mathrm{R}}^{-}$, alors,

$$
K(t) \simeq\left(1 / \tau^{2}\right) \exp -(t / \tau)
$$

$$
\begin{aligned}
\varphi(t) \simeq\left\{\cos \left(\frac{\sqrt{3}}{2} \frac{t}{\tau}\right)+\frac{1}{\sqrt{3}} \sin \left(\frac{\sqrt{3}}{2} \frac{t}{\tau}\right)\right\} & \\
& \times \exp -(t / 2 \tau) .
\end{aligned}
$$

5. Conclusion. - Nos résultats expérimentaux mettent en évidence l'existence d'une anisotropie de l'ion hydraté ; l'interprétation que nous en donnons permet de répartir les ions étudiés en 4 catégories selon l'importance de cette anisotropie.

\section{Bibliographie}

[1] Bellissent, M. C., Gérard, P., Longevialle, C., Meton, M., Pich, M., Morand, G., J. Electrochem. Soc. 118 (1972) 1944.

[2] Wind, C. H., Versl. Kon. Akad. Ams. 3 (1894) 82 et 4 (1895) 210.

[3] Holzapfel, Z., Phys. Chem. N.F. Bd 59(1968) S 166.

[4] Picard, E. J., Thèse Paris (1965).

[5] Friedman, H. L., J. Chim. Phys. 19e Réunion de la Société de Chimie Physique Montpellier 1968, No spécial Oct. 1969, 75. 